\title{
ALPORT SYNDROME: SIGNIFICANCE OF GINGIVAL BIOPSY IN THE INITIAL DIAGNOSIS AND PERIODONTAL EVALUATION AFTER RENAL TRANSPLANTATION
}

\author{
Hilal Uslu TOYGAR ${ }^{1}$, Okan TOYGAR ${ }^{2}$, Esra GUZELDEMIR ${ }^{3}$, Ulkem CILASUN ${ }^{4}$, Ahmet NACAR ${ }^{5}$, Nebil BAL
}

\author{
1 - DDS, Ph.D, Assistant Professor, Department of Periodontology, Faculty of Dentistry, Baskent University, Ankara, Turkey. \\ 2 - MD, Private Practice, Adana, Turkey. \\ 3 - DDS, Ph.D, Department of Periodontology, Faculty of Dentistry, Baskent University, Ankara, Turkey. \\ 4 - DDS, Ph.D, Assistant Professor, Department of Oral and Maxillofacial Surgery, Faculty of Dentistry, Kocaeli University, Kocaeli, Turkey \\ 5 - MD, Associate Professor, Department of Pathology, Faculty of Medicine, Mustafa Kemal University, Antakya, Hatay, Turkey. \\ 6 - MD, Associate Professor, Department of Pathology, Faculty of Medicine, Baskent University, Ankara, Turkey.
}

Corresponding address: Hilal Uslu Toygar, DDS, Ph.D - Baskent Universitesi - Adana Uygulama ve Arastirma Hastanesi - Agiz ve Dis Sagligi Klinigi - Kazim Karabekir Mah, 59 Sok. No 91 - Yuregir, 01150 Adana - Turkey - Phone: +90 322322 8282/1081 - Fax: +90 3223227979 - e-mail: usluhilaladana@yahoo.com

Received: July 10, 2008 - Modification: November 30, 2008 - Accepted: April 23, 2009

\begin{abstract}
A lport Syndrome (AS) is an important hereditary disorder affecting the glomerular basement membrane. Diagnosis of AS is based on the presence of hematuric nephropathy, renal failure, hearing loss, ocular abnormalities and changes in the glomerular basement membrane of the lamina densa. The aims of this case report were to show the changes in the gingival tissues in a patient with AS under therapy with cyclosporin-A after renal transplantation and to discuss the possible role of type IV collagen in gingival basal lamina as an alternative approach for the diagnosis of AS. A 20-year-old male patient with AS underwent periodontal therapy including a series of gingivectomy surgeries. Gingival samples obtained during the second surgery were examined histopathologically and by transmission electron microscopy for further pathological examination. Gingivectomy procedures have been performed every 6 months over the last 4 years. The excessive and fibrous gingival enlargements resulted in migration of the anterior teeth, but no alveolar bone loss occurred. This is the first report to demonstrate the possible changes in the gingival tissues caused by AS. It is suggested that gingival biopsy can be an initial diagnostic tool instead of renal or skin biopsies. Proper dental and periodontal care and regular visits to the dentist could provide limited gingival hyperplasia to patients with AS.
\end{abstract}

Keywords: Alport syndrome. Hereditary nephritis. Gingival overgrowth. Biopsy. Kidney transplantation. Cyclosporin

\section{INTRODUCTION}

Alport Syndrome (AS) was first described in 1927 as a hereditary disorder characterized by a combination of nephritis and deafness ${ }^{1}$. This syndrome is a basement membrane (BM) disease affecting approximately 1 in every 5,000 people ${ }^{7}$. The disease manifests with juvenile to adult onset progressive glomerulonephritis usually associated with a high-frequency-specific sensorineural hearing loss, dot and fleck retinopathy, and lens abnormalities ${ }^{12}$. Two forms have been recognized in the molecular genetic base of AS. The dominant X-linked form owing to mutations of locus COL4A5 and the recessive autosomal form resulting from mutations to loci COL4A3 or COL4A43. However, the genealogical analysis suggests a dominant autosomal type. Although approximately $85 \%$ of the cases are Xlinked dominant form there is also a recessive autosomal form for about $15 \%$. Family history is negative in isolated cases of de novo mutation.

Type IV collagen is the main component of the basal membrane. The mutation presented in AS produces defects in chains $\alpha 3, \alpha 4$, and $\alpha 5$ (IV). Type IV collagen damage, owing to mutation, breaks the epithelial bonds and leads to an organ defect ${ }^{14}$. These defects in chains result in entanglement and incorrect set-up of monomers which are quickly degraded. These mutations interrupt the normal replacement of the embryonic development and cause persistence of chains $\alpha 1 . \alpha 1 \alpha 2$ (IV) in renal basal membranes, cochlea and capsule of lenses.

The main structural and fibrilar component of the human gingival connective tissue is collagen, which accounts for about $60 \%$ of the total tissue protein. Previous biochemical studies have demonstrated that type IV collagen accounts for less than $1 \%$ of the total collagenous component of the healthy human gingival connective tissue $^{20}$. Type IV collagen is presented at the BM of the 
human gingiva, which separates the connective tissue from the epithelium.

The clinical presentation of the syndrome emerges with signs such as; hematuria, progressive renal failure, sensorineural hearing loss (SNHL) (variable intensity, bilateral, symmetrical and progressive; present in approximately $55 \%$ of men and $45 \%$ of women), ocular affections, (anterior lenticonus, ocular stains and cataracts $^{4}$ ), macro-thrombocytopenia ${ }^{23}$ and leiomyomatosis ${ }^{27}$.

Hearing loss is one of the initial and most common sign in a patient with hematuria. SNHL in high frequencies suggests AS even in the absence of renal biopsy or family history of renal failure. It is generally accepted that the initial approach to a patient with clinical features and positive family history of AS is skin biopsy and the presence of $\alpha 5$ collagen chain in the skin leads to renal biopsy ${ }^{16}$. Renal biopsy may be risky in cases of renal failure ${ }^{11,16,17,23,27}$. Careful follow-up of the hearing loss is a relevant factor of the prognosis of the renal disease progression. Men with hearing loss in the first two decades of life have more severe renal diseases ${ }^{11}$. The regular assessment of hearing in a patient with AS is very important. Ocular abnormalities in these patients hinder visual clues which are essential for the communication with the patient who has severe hearing loss.

Dialysis and renal transplantation are indicated in this group of patients with AS and end-stage renal failure ${ }^{12,17}$. Immunosuppressant drugs, cyclosporin-A (CsA) and corticoids are administered to patients who undergo renal transplantation. The prognosis improves with renal transplantation increasing longevity of the patient with AS and renal failure ${ }^{12}$.

The aims of this case report are to show the changes in the gingival tissues in a patient with AS under therapy with cyclosporin-A after renal transplantation and to discuss the possible role of type IV collagen in gingival basal lamina as an alternative approach for the diagnosis of AS.

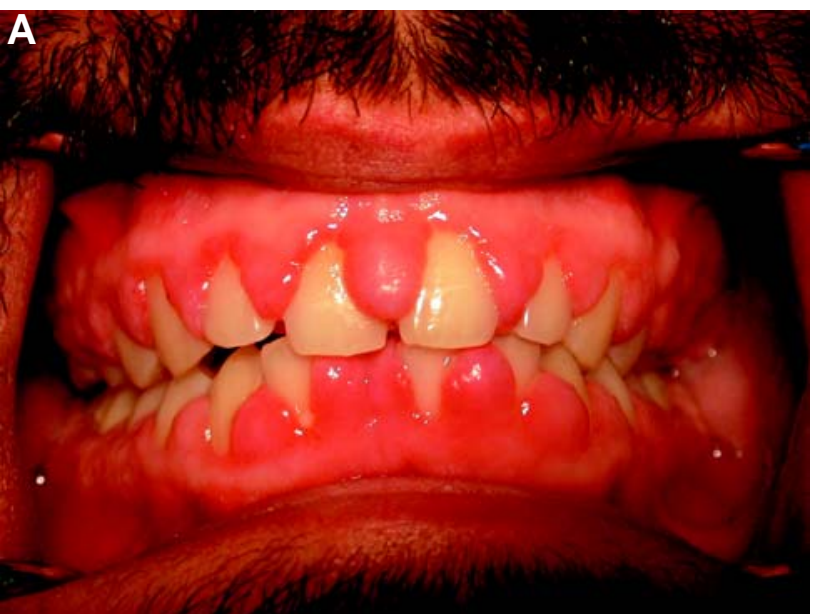

\section{CASE REPORT}

\section{Case Description and Gingival Surgery}

In 2003, a 20-year-old male patient was referred to the Baskent University, Faculty of Dentistry, Department of Periodontology, Ankara, Turkey for treatment of severe gingival hyperplasia. The patient's persistent microhematuria was discovered when he was 3 years old during a routine pediatric examination. While he had temporary bilateral hearing loss, no abnormalities were seen in the optical media, specifically no cataract or lenticonus. There was no family history. He had a renal biopsy at the University of Heidelberg in Germany and he was diagnosed as having AS. His family history was also negative to de novo mutation.

Review of medical history revealed that at the age of 16 , the patient started a regular hemodialysis regimen due to the end-stage renal disease. He was scheduled for renal transplantation and was put under an immunosuppressive protocol in the meantime. The patient received a kidney from his mother and, after the transplantation, immunosuppressant drugs - cyclosporin-A ( $5 \mathrm{mg} / \mathrm{kg} /$ day), prednisolone $(15 \mathrm{mg} / \mathrm{kg} /$ day $)$ and azathioprine $(2 \mathrm{mg} / \mathrm{kg} /$ day) - were prescribed for prevention of transplant rejection. His postoperative healing period was uneventful and he had a good renal function with creatinine levels ranging from 0.9 to $2.9 \mathrm{mg} / \mathrm{dL}$ at present. No rejection episode was occurred.

He had progressive and symmetrical SNHL hearing loss, mainly affecting high frequency ranges $(>2,000 \mathrm{~Hz})$. He had bilateral cochlear implants for the rehabilitation of hearing function. At the age of 22, bilateral visual failure began because of a large myopic shift. On the ophthalmologic examination, his visual acuity was 20/100 with $-4.00(-4.50 * 120)$ in the right eye and 20/70 with $4.25(-2.50 * 170)$ in the left eye. The ocular surface, cornea and anterior chamber were normal. Slit lamp examination revealed anterior lenticonus in both eyes. Both lenses were clear with no evidence of cataract. Fundus examination was normal in both eyes. Right eye underwent a clear lens phacoemulsification surgery and intraocular lens

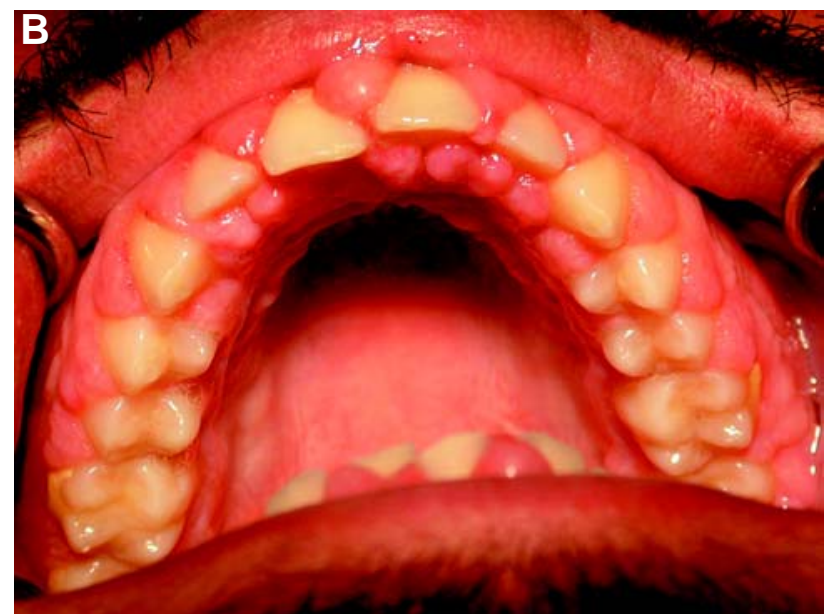

FIGURE 1- Gingival enlargement affecting the buccal (A) and palatal (B) surfaces of all teeth 

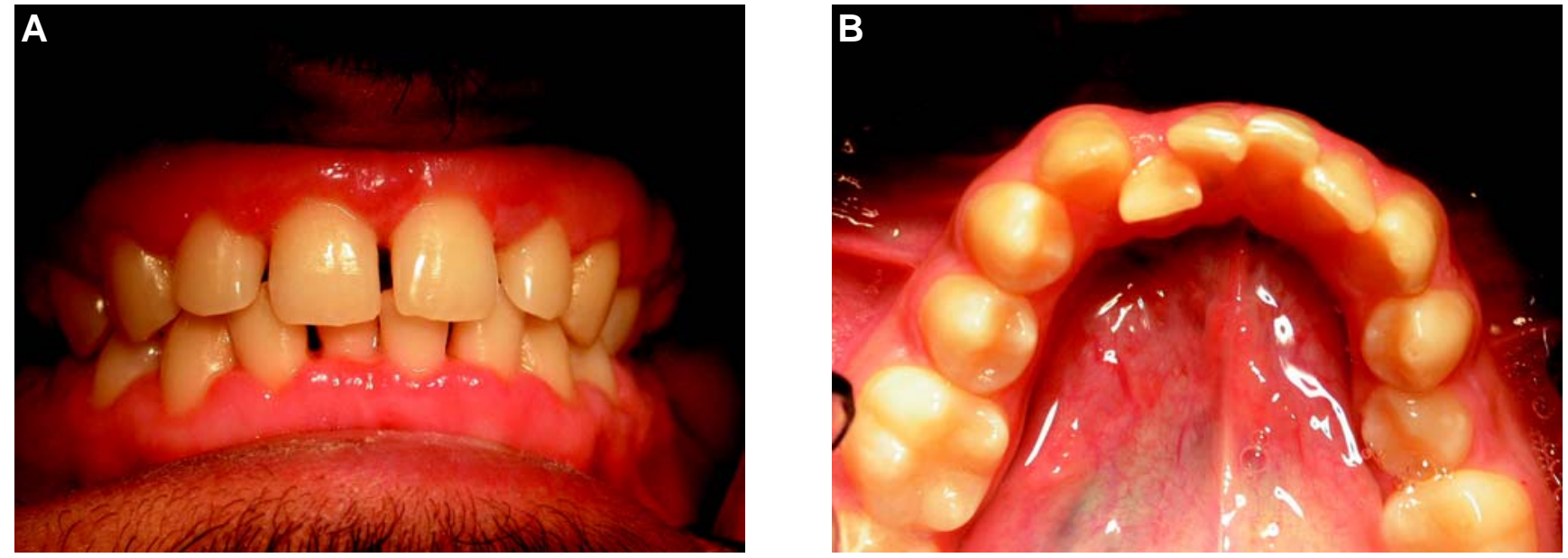

FIGURE 2- Gingivectomy performed at the buccal (A) and lingual (B) surfaces

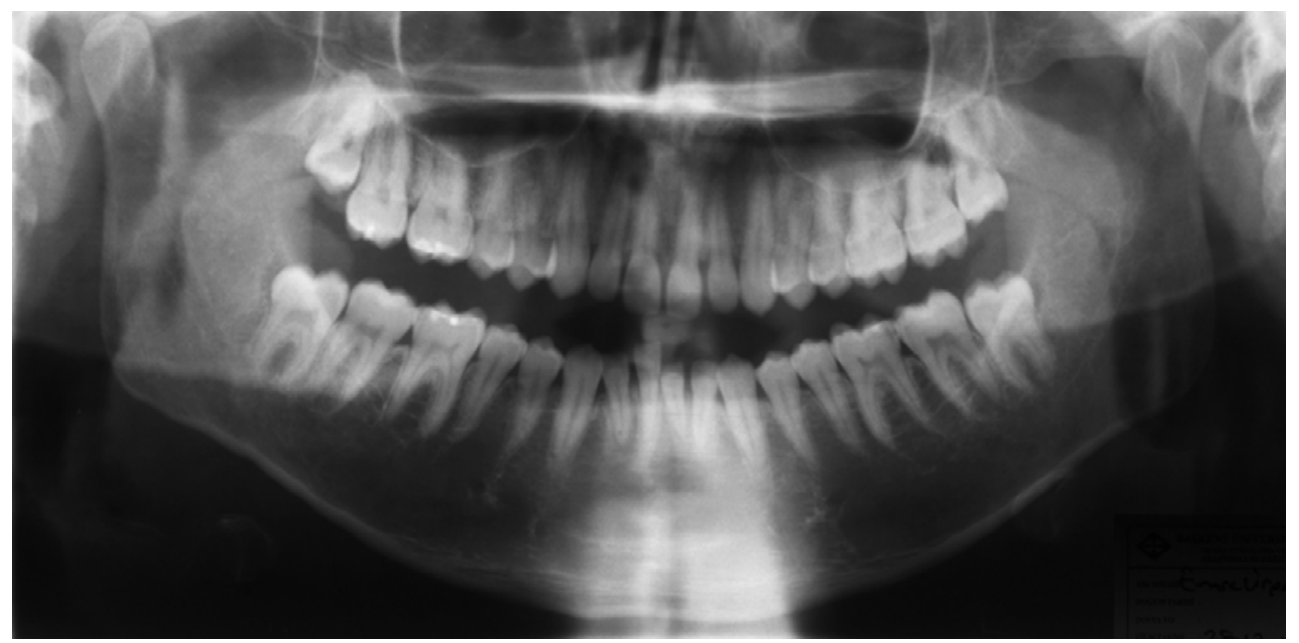

FIGURE 3- Migration of anterior teeth due to excessive and fibrous gingival enlargement, though without alveolar bone loss

implantation. After the surgery, visual acuity increased to $20 / 30$ with $+0.50(-2.00 * 10)$ in right eye. Similar procedure has planned for the left eye.

One year after renal transplantation, the patient started complaining of gingival enlargement affecting both buccal and lingual/palatal surfaces of all teeth (Figure 1). Although his oral hygiene was poor, he had no decayed or missing teeth. Gingival enlargement treatment started with oral hygiene instructions and scaling and root planning. First gingivectomy surgery was performed at the anterior regions of both maxilla and mandible with antibiotic prophylaxis ( $2 \mathrm{~g}$ amoxicillin/30 min before surgery), to recover esthetic. Two weeks later, gingivectomy was performed in all posterior regions. Wound healing was uneventful (Figure 2 ). Four months later, however, recurrence of gingival enlargement was observed at all gingival sites. Gingival biopsy was taken for histopathological analysis by light microscopy and immunohistochemistry and examination by transmission electron microscopy (TEM).

For histopathological analysis, gingival biopsies were done and the specimens were fixed in $10 \%$ formalin solution and embedded in paraffin blocks after routine processing. Then, 4-5- $\mu \mathrm{m}$-thick sections were obtained and stained with hematoxylin and eosin (HE). Microscopic examination of the HE-stained slides showed epithelial hyperplasia and fibrosis. Serial sections were immunostained with a biotin-streptavidin complex system (ACE+ Substrate chromogene Ready to Use, K3469, DAKO, Denmark) for type IV collagen (Clone CIV 22, Nr. M 0785 USA) and the basal membrane under the squamous epithelium was observed on some sites with discontinuity (Figure 4).

For TEM analysis, the gingival specimens were fixed in phosphate-buffered saline containing $2.5 \%$ glutaraldehyde for $2-3 \mathrm{~h}$, post-fixed in $1 \%$ osmium tetroxide and dehydrated in a series of increasing ethanol concentrations $(25 \%, 50 \%, 75 \%, 95 \%$, and absolute alcohol). After passing through propylene oxide, the specimens were embedded in quick-setting epoxy resin (Araldite CY 212, Ciba-Geigy, Basel, Switzerland) DDSA (2-dodecenyl succinic anhydride), BDMA (benzyldimethylamine) and dibutylphtalate. Semithin sections were obtained and stained with blue toluidin and examined with a light microscope. Ultrathin sections were 


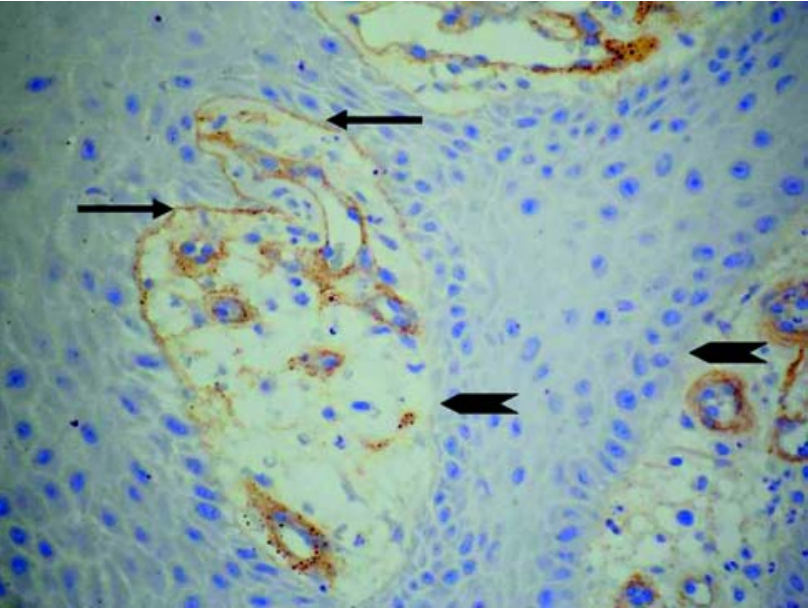

FIGURE 4- Epithelial hyperplasia and fibrosis observed under light microscopy and immunohistochemistry with type IV collagen staining. Basal membrane under the squamous epithelium is observed on some sites with discontinuity (arrows) (x400)

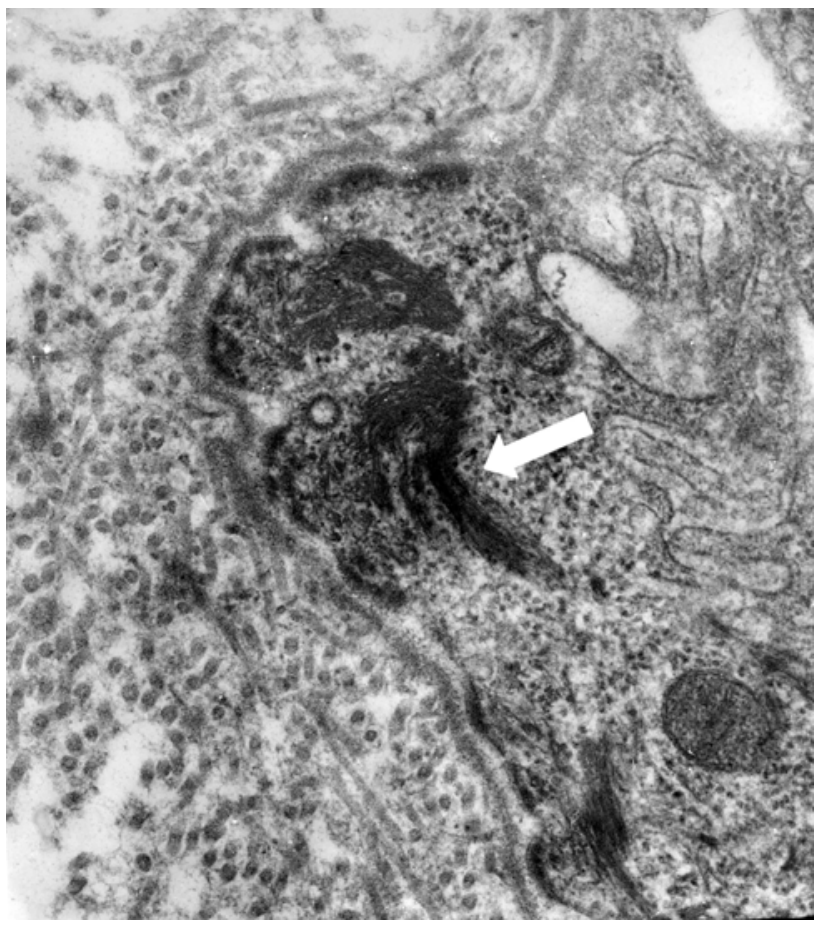

FIGURE 5- TEM micrograph showing tonofilament clumping (arrow) (x27800)

stained with uranyl acetate and lead citrate and examined with LEO 906E EM transmission electron microscope (Zeiss, Jena, Germany). There was tonofilament accumulation in most epithelial basal cells (Figure 5). The lamina densa was disrupted in several regions (Figures 6 and 7) and hemidesmosoms remained intact (Figure 8). Dense bundles of thick collagen fibers were organized around and under the BM (Figure 9).

In order to protect the patient from the side effects of CsA therapy on the gingiva tissues, the patient was referred

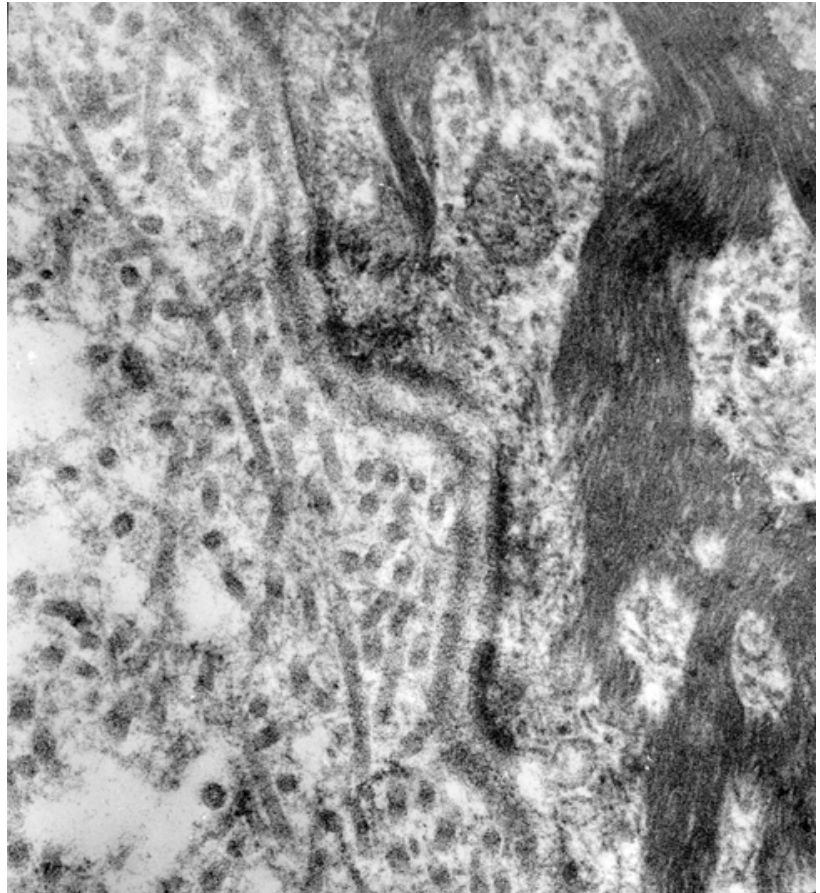

FIGURE 6- TEM micrograph showing basement membrane (lamina densa; darker line, lamina lucida; lighter line) and tonofilaments (x46460)

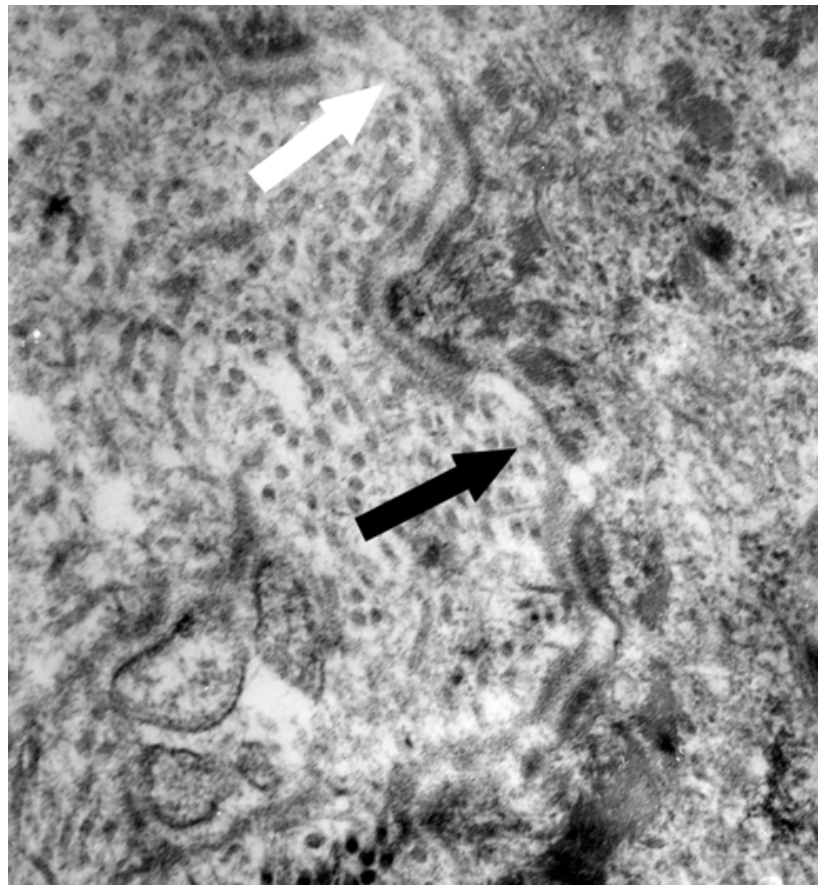

FIGURE 7-TEM micrograph showing disrupted (white arrow) and discontinuous (black arrow) basement membrane (x35970)

to the Nephrology Department for consultation. However, the nephrologist pointed out that the patient had an aggressive course of AS and that, since control of the disease was accomplished successfully with CsA, he was not willing to change the medication. 


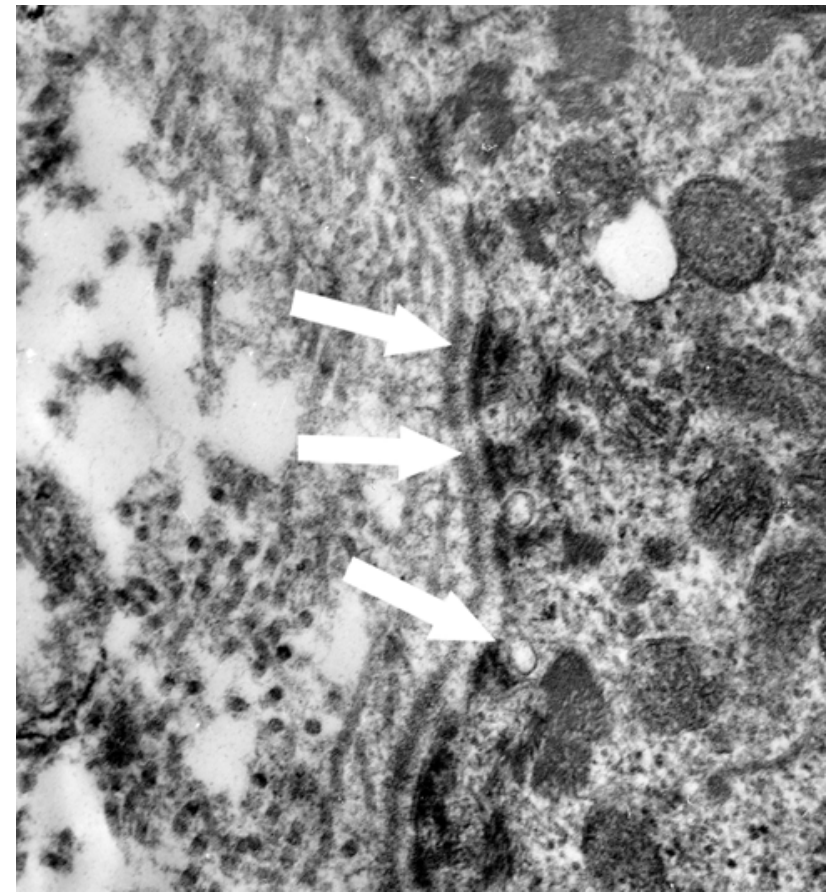

FIGURE 8- TEM micrograph showing intact hemidesmozoms (arrows) (x35970)

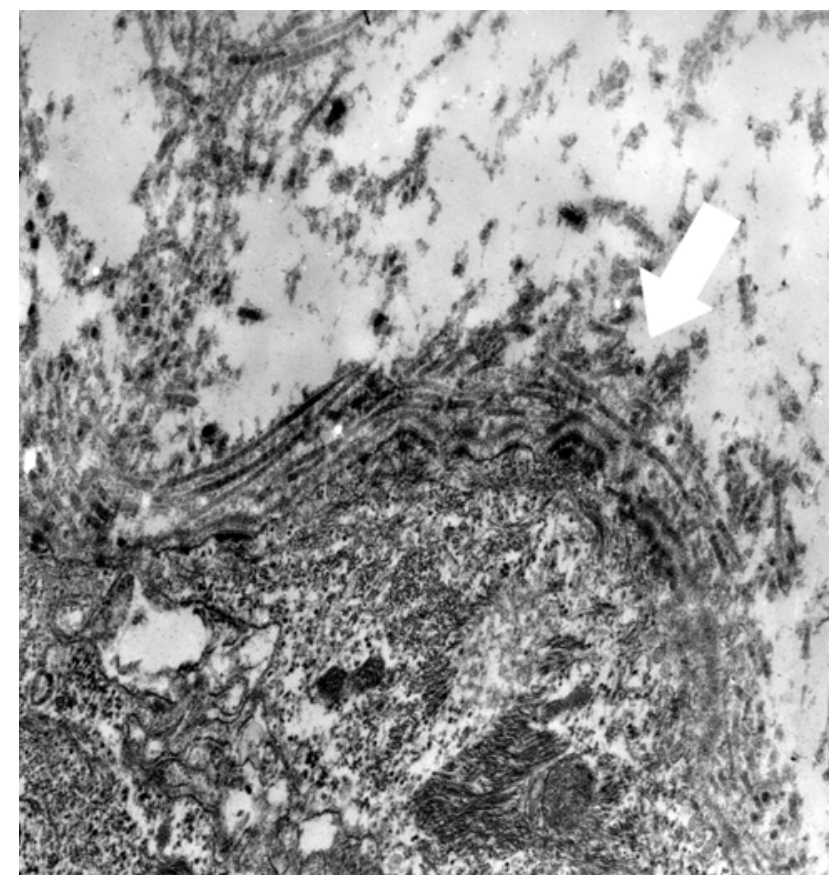

FIGURE 9- TEM micrograph showing collagen fibers organized parallel bundles under the basement membrane (x16700)

Gingivectomy procedures have been performed every 6 months during the last 4 years. The excessive and fibrous gingival enlargements resulted in migration of the anterior teeth, but no alveolar bone loss occurred (Figure 3 ).

\section{DISCUSSION}

AS is a hereditary disorder characterized by hematuric nephritis leading to an end-stage renal disease, sensoryneural deafness and ocular abnormalities. AS have been attributed to the production of a defective type IV collagen ${ }^{21}$. Type IV collagen is the main component of the glomerular $\mathrm{BM}$ and composed of six genetically distinct chains, namely $\alpha 1$ (IV) - $\alpha 6$ (IV), encoded by gene pairs located on three different chromosomes ${ }^{10,20,26}$.

Renal transplantation has proven to be the best treatment for patients with an end-stage renal disease ${ }^{18,24}$. It is generally successful in patients with AS but in cases where graft loss occurs, re-transplantation usually fails. CsA has been shown to be effective at halting the progression of renal failure in $\mathrm{AS}^{2}$. CsA is a cyclic polypeptide with potent and selective immunosuppressive activity, acting primarily by inhibiting interleukin-2 gene transcription in CD4+ lymphocytes ${ }^{6}$. CsA treatment is associated with side effects including chronic nephrotoxicity ${ }^{30}$, hepatotoxicity ${ }^{9}$ and neurotoxicity ${ }^{3}$, hypertention ${ }^{27,28}$ and gingival overgrowth ${ }^{5}$.

The patient of this case received a kidney from his mother and was put in an immunosuppressive drug regimen with CsA $(5 \mathrm{mg} / \mathrm{kg} / \mathrm{d})$, prednisolone $(15 \mathrm{mg} / \mathrm{kg} / \mathrm{d})$, and azathioprine $(2 \mathrm{mg} / \mathrm{kg} / \mathrm{d})$. The prednisolone dose was gradually reduced to $10 \mathrm{mg} / \mathrm{kg} / \mathrm{d}$. The relationship between CsA and the development of gingival overgrowth is well established in humans as well as in animal experimental models ${ }^{5,8}$. Gingival enlargement, occurring in a range of 25$81 \%$ of the patients, is a severe pathology that interferes with normal oral functions such as mastication and speech, and causes profound psychological problems to the patients ${ }^{8}$. Hyland, et al. ${ }^{15}$ (2003) recently suggested that since less 50\% of the patients taking CsA develop gingival overgrowth, a genetic predisposing may explain the different responses of gingival fibroblasts in collagen turnover regulation after CsA treatment and may play a major role in the pathogenesis of CsA-induced overgrowth. Other factors such as the influence of different pre-existing gingival conditions (e.g. gingival inflammation) and the different host's capacity to deal metabolically with CsA should also be considered as they might affect CsA-induced pathogenesis ${ }^{8}$.

Collagen comprises more than $60 \%$ of the periodontal tissues. The basal lamina, which is largely composed of glycoproteins (laminin and nidogen), collagen type IV, and proteoglycans, underlies the oral epithelium and forms a barrier between the epithelium and the connective tissue. Interactions between epithelial cells and fibroblasts stimulate type IV collagen and laminin expression in epithelial cells in vitro ${ }^{25}$. Type IV collagen was observed immunohistochemically underneath the basal epithelial cells $^{29}$. These molecules are important in epithelium formation and they establish the junctional seal separating the oral cavity from the interstitial environment. A breakdown of this seal is a significant factor in periodontal disease development and its reformation is required for successful periodontal treatment or periodontal 
regeneration ${ }^{21}$. AS is caused by defects in type IV collagen, which is the major component of BM. It is generally accepted that the initial approach for patients with the clinical features and a sustaining family history of AS is skin biopsy. In the presence of the $\alpha 5$ collagen chain in the skin, next approach would be a renal biopsy ${ }^{25}$. The typically thin glomerular BM thickens over time into multilamellations surrounding lucent areas that often contain granules of varying densities ${ }^{13}$.

The diagnostic value of skin biopsy has first described in 1986 since a kidney biopsy could be risky in cases of renal failure ${ }^{22}$. Skin biopsy is especially useful if a kidney biopsy poses an excessive risk, as in the patients with an end-stage renal disease. While the $\alpha 5$ chain of type IV collagen is also expressed in epidermis, an immunofluorescence examination of skin biopsy specimen can be used to establish the diagnosis. Approximately $80 \%$ of male patients and $60 \%$ of female patients with X-linked AS have no $\alpha 5(\mathrm{IV})$ collagen in epidermal BM. In addition, most individuals with autosomal recessive AS do not express $\alpha 3$ (IV), $\alpha 4$ (IV), or $\alpha 5$ (IV) collagens in skin. Healthy individuals and patients with thin-membrane disease have normal expression of $\alpha 5$ (IV) in the skin. Hamiwka, et al. ${ }^{13}$ (2007) suggested that when a kidney biopsy is considered necessary in a female patient with persistent hematuria but without a clear family history of AS or proteinuria, renal biopsy alone may not be enough to exclude AS carrier status. A skin biopsy obtained at the time of the kidney biopsy should not pose any further risk for the patient but can provide some additional information.

Could gingiva, which has type IV collagen in it is BM be valuable for the diagnosis of AS? In this report, immunohistochemical analysis revealed disrupted areas on the BM. These findings were supported by TEM analysis, which showed that the lamina densa was disrupted in several regions of the BM. In the gingival tissue, the basal lamina is largely composed by collagen type IV. The reason of these irregularities and disruptions might have been arisen from type IV collagen.

Gingival biopsy is easily done under local anesthesia, and tissue specimens that are obtained from the intraoral region do not cause esthetic problems.

In addition to having AS, our patient was an organ transplant recipient and under immunosuppressive therapy with CsA. The effects of CsA on gingiva have been recognized but not many studies have discussed the effects of CsA on type IV collagen are available. Mondella, et al. ${ }^{19}$ (1994) evaluated the BM changes in a group of patients with psoriasis and consuming CsA. In the patients with discontinuous staining of type IV collagen, evident and continuous staining of type IV collagen within the BM was observed after the CsA treatment.

The results of the present case indicates that when the effect of CsA on the repair of the continuity of type IV collagen in the BM is considered, despite the CsA therapy, the continuing irregularity and discontinuity in the basal lamina increases the possibility of gingival type IV collagen defects in the patients with AS.

There is no evidence about the possible changes that AS causes in the gingival tissues. Therefore the precise evaluation of the effects of CsA on the gingival tissues of the patients with AS is not possible. Future studies should evaluate the effects of AS on type IV collagen in gingiva and the influence of CsA, which has an important role in AS both pre and post-transplantation, on the gingival collagen.

Patients with AS and renal failure have their prognosis improved and their longevity increased with renal transplantation. Therefore, the follow up, rehabilitation, and investigation of the progression of hearing loss and ophthalmological problems become important in the quality of life of these patients. Maintenance of good oral hygiene with regular visits to the dentist can limit gingival hyperplasia. As in all patients under immunosuppressive treatment, prevention of infection is also very important for patients with AS. CsA has a determinant role before and after renal transplantation as well as on the treatment of AS, and the side effects of this drug on gingiva are increased in the presence of infection. Aggressive and severe gingival hyperplasia hinders biofilm control and causes migration of teeth. If untreated, this condition can compromise the patients' dental and gingival esthetics and cause nutrition problems, and periodontal or dental infections.

In order to prolong the lifetime, alongside with regular nephrological, ocular and hearing evaluations, periodical examinations of the oral tissues are consequent for these patients with a low quality of life. The possible role of gingival biopsy, which is easier and less risky than renal or skin biopsies, on the early diagnosis and evaluations of the treatment results of AS should be investigated.

\section{REFERENCES}

1- Alport A. Hereditary familial congenital haemorrhagic nephritis. Brit Med J. 1927;1:504-6.

2- Callis L, Vila A, Carrera M, Nieto J. Long-term effects of cyclosporine A in Alport's syndrome. Kidney Int. 1999;55(3):1051-6.

3- Chang SH, Lim CS, Low TS, Chong HT, Tan SY. Cyclosporineassociated encephalopathy: a case report and literature review. Transplant Proc. 2001;33(7-8):3700-1.

4- Choi J, Na K, Bae S, Roh G. Anterior lens capsule abnormalities in Alport syndrome. Korean J Ophthalmol. 2005;19(1):84-9.

5- Dongari A, McDonnell HT, Langlais RP. Drug-induced gingival overgrowth. Oral Surg Oral Med Oral Pathol. 1993;76(4):543-8.

6- Faulds D, Goa KL, Benfield P. Cyclosporin. A review of its pharmacodynamic and pharmacokinetic properties, and therapeutic use in immunoregulatory disorders. Drugs.1993;45(6):953-1040.

7- Flinter F, Bobrow M. The molecular genetics of Alport syndrome: report of two workshops. J Med Genet. 1992;29(5):352-3.

8- Gagliano N, Moscheni C, Dellavia C, Torri C, Stabellini G, Ferrario $\mathrm{VF}$, et al. Effect of cyclosporin A on human gingival fibroblast collagen turnover in relation to the development of gingival overgrowth: an in vitro study. Biomed Pharmacother. 2004;58(4):231-8.

9- Galan AI, Fernandez E, Moran D, Munoz ME, Jimenez R. Cyclosporine A hepatotoxicity: effect of prolonged treatment with cyclosporine on biliary lipid secretion in the rat. Clin Exp Pharmacol Physiol. 1995;22(4):260-5. 
10- Gross O, Netzer KO, Lambrecht R, Seibold S, Weber M. Meta-analysis of genotype-phenotype correlation in X-linked Alport syndrome: impact on clinical counselling. Nephrol Dial Transplant. 2002;17(7):1218-27.

11- Gubler MC, Heidet L, Antignac C. Alport syndrome or progressive hereditary nephritis with hearing loss. Nephrol Ther. 2007;3(3):113-20.

12- Haberal M, Emiroglu R, Mahmutoglu M, Karakayali H, Turan M, Erdal R. Alport's syndrome and renal transplantation. Transplant Proc. 2001;33(5):2702-3

13- Hamiwka LA, George DH, Grisaru S, Midgley JP. Discordance between skin biopsy and kidney biopsy in an X-linked carrier of Alport syndrome. Pediatr Nephrol. 2007;22(7):1050-3

14- Hudson BG, Tryggvason K, Sundaramoorthy M, Neilson EG. Alport's syndrome, Goodpasture's syndrome, and type IV collagen. N Engl J Med. $2003 ; 348(25): 2543-56$

15- Hyland PL, Traynor PS, Myrillas TT, Marley JJ, Linden GJ, Winter P, et al. The effects of cyclosporin on the collagenolytic activity of gingival fibroblasts. J Periodontol. 2003;74(4):437-45.

16- Kashtan CE. Alport syndrome. An inherited disorder of renal, ocular, and cochlear basement membranes. Medicine (Baltimore). 1999;78(5):338-60.

17- Kashtan CE. Renal transplantation in patients with Alport syndrome. Pediatr Transplant. 2006;10(6):651-7.

18- Milliner DS, Pierides AM, Holley KE. Renal transplantation in Alport's syndrome: anti-glomerular basement membrane glomerulonephritis in the allograft. Mayo Clin Proc. 1982;57(1):35-43.

19- Mondello MR, Califano L, Cannavo SP, Di Mauro D, Guarneri B, Magaudda L, et al. Psoriasis and cyclosporin: immunohistochemical aspects of the basement membrane. Acta Derm Venereol Suppl. (Stockh). 1994; 186:96-8

20- Narayanan AS, Page RC. Connective tissues of the periodontium: a summary of current work. Coll Relat Res. 1983;3(1):33-64.

21- Palaiologou AA, Yukna RA, Moses R, Lallier TE. Gingival, dermal, and periodontal ligament fibroblasts express different extracellular matrix receptors. J Periodontol. 2001;72(6):798-807.

22- Pirson Y. Making the diagnosis of Alport's syndrome. Kidney Int. 1999;56(2):760-75

23- Richardson D, Shires M, Davison AM. Renal diagnosis without renal biopsy. Nephritis and sensorineural deafness. Nephrol Dial Transplant. 2001;16(6):1291-4

24- Sefer S, Trotic R, Lacmanovic V, Degoricia V, Ratkovic-Gusic I, Kes P. Effects of renal transplantation on hearing and ocular changes in a monozygotic twin with Alport's syndrome: comparison with other twin on hemodialysis. Croat Med J. 2000;41(2):203-6.

25- Shimonishi M, Sato J, Takahashi N, Komatsu M. Expression of type IV collagen and laminin at the interface between epithelial cells and fibroblasts from human periodontal ligament. Eur J Oral Sci. 2005; $113(1): 34-40$

26- Slajpah M, Meglic A, Furlan P, Glavac D. The importance of noninvasive genetic analysis in the initial diagnosis of Alport syndrome in young patients. Pediatr Nephrol. 2005;20(9):1260-4.

27- Sugimoto K, Yanagida H, Yagi K, Kuwajima H, Okada M, Takemura T. A Japanese family with Alport syndrome associated with esophageal leiomyomatosis: genetic analysis of COL4A5 to COL4A6 and immunostaining for type IV collagen subtypes. Clin Nephrol. 2005;64(2):144-50.
28- Textor SC, Canzanello VJ, Taler SJ, Wilson DJ, Schwartz LL, Augustine JE, et al. Cyclosporine-induced hypertension after transplantation. Mayo Clin Proc. 1994;69(12):1182-93.

29- Tomakidi P, Fusenig NE, Kohl A, Komposch G. Histomorphological and biochemical differentiation capacity in organotypic co-cultures of primary gingival cells. J Periodontal Res. 1997;32(4):388-400.

30- Woolfson RG, Neild GH. Cyclosporin nephrotoxicity following cardiac transplantation. Nephrol Dial Transplant. 1997;12(10):2054-6. 